\title{
INTERPOLATION METHODS OF CONSTANTS AND MEANS WITH QUASI-POWER FUNCTION PARAMETERS
}

\author{
MING FAN
}

\begin{abstract}
The purpose of this paper is to describe the interpolation methods of constants and means with quasi-power function parameters in the sense of Brudnyi-Krugljak's $K$ - and $J$-methods with the weighted Orlicz space parameters. As an application, the relationship of such methods with particular parameters and the complex methods for Banach spaces of Fourier type is sharpened.
\end{abstract}

\section{Introduction and main theorem}

An interesting problem in interpolation theory is to find the connection between interpolation methods whose definitions are considerably different. It is known that the generalized Lions-Peetre methods of constants and means are equivalent to the real $K$ - and $J$-methods due to Brudnyi-Krugljak with certain parameters [2, Th.4.2.11 \& Th.4.2.33], but such parameters were only calculated for the classical real methods [1, Th.3.12.1]. In the present paper, we intend to determine explicitly the interpolation methods of constants and means with quasi-power function parameters in the sense of Brudnyi-Krugljak's methods with the weighted Orlicz space parameters. This paper is ordered as follows: Section 1 contains basic definitions and the main theorem. The proof of the main theorem will be presented in the next three sections. In the final two sections, we study the relationship of this kind of real methods with a family of particular function parameters and the complex methods for Banach spaces of Fourier type, as well as some related topic concerning the non-commutative $L^{1}$-spaces.

We begin with some basic definitions and terminology in the interpolation theory that we will use. The notations $\subseteq$ and $=$ beween Banach spaces stand for continuous inclusion and isomorphic equivalence respectively. By a Banach couple we mean a pair of Banach spaces $\bar{X}=\left(X_{0}, X_{1}\right)$ over the complex field C if both $X_{0}$ and $X_{1}$ are continuously imbedded in some Hausdorff to- 
pological vector space, and set $\Delta \bar{X}=X_{0} \cap X_{1}$ and $\Sigma \bar{X}=X_{0}+X_{1}$ with the norms $\|x\|_{\Delta \bar{X}}=\|x\|_{0} \vee\|x\|_{1}$ for $x \in \Delta \bar{X}$ and

$$
\|x\|_{\Sigma \bar{X}}=\inf \left\{\left\|x_{0}\right\|_{0}+t\left\|x_{1}\right\|_{1} \mid x=x_{0}+x_{1} \text { with } x_{j} \in X j\right\} \quad \text { for } x \in \Sigma \bar{X} \text {. }
$$

A Banach space $X$ is called an intermediate space for $\bar{X}$ if $\Delta \bar{X} \subseteq X \subseteq \Sigma \bar{X}$. The regulation $X^{0}$ of $X$ for $\bar{X}$ is the closure of $\Delta \bar{X}$ in $X$, and the dual space $X^{\prime}$ of $X$ for $\bar{X}$ is the dual space of $X^{0}$ in the usual sense. An intermediate space $X$ for $\bar{X}$ is called an interpolation space if every bounded linear operator $T=\left(T_{0}, T_{1}\right)$ on $\bar{X}$, where $T_{j}$ is a bounded linear operator on $X_{j}(j=0,1)$ with $T_{0}\left|X_{0}=T_{1}\right| X_{1}$, produces a bounded linear operator on $X$. See [1] and [2] for further details.

For $a, b>0$, we denote $a \vee b=\max \{a, b\}$ and $a \wedge b=\min \{a, b\}$. For positive functions $f$ and $g$ defined in $\mathrm{R}^{+}$, where $\mathrm{R}^{+}=(0, \infty)$, we write $f \approx g$ if they are equivalent in the sense that $\exists c_{1}, c_{2}>0$ with $c_{1} g(t) \leq f(t) \leq c_{2} g(t)$ for all $t>0$. Here and throughout, we assume that $1 \leq p_{0}, p_{1} \leq \infty$ with $p_{0} \neq p_{1}$ (unless specified otherwise), and that $\rho: \mathrm{R}^{+} \rightarrow \mathrm{R}^{+}$is a quasi-power function in the sense that for some $\alpha \in(0,1)$ and $c>0$ we have

$$
\rho(\lambda t) \leq c\left(\lambda^{\alpha} \vee \lambda^{1-\alpha}\right) \rho(t) \quad \forall \lambda>0, t>0 .
$$

By replacing with an equivalent function, we can assume that $\rho$ is concave with $\rho(1)=1$ and

$$
\rho(\lambda t) \leq\left(\lambda^{\alpha} \vee \lambda^{1-\alpha}\right) \rho(t) \quad \forall \lambda>0, t>0 .
$$

Now we define the generalized Lions-Peetre methods of constants $K_{\rho, p_{0}, p_{1}}$ and methods of means $J_{\rho, p_{0}, p_{1}}$, which agree with [2, Def.4.2.9 \& (4.2.65)] in the special case:

Definition 1.1. For a Banach couple $\bar{X}=\left(X_{0}, X_{1}\right)$, let $K_{\rho, p_{0}, p_{1}}(\bar{X})$ be the space of all those $x \in \Sigma \bar{X}$ such that there exist strongly measurable functions $x_{j}: \mathrm{R}^{+} \rightarrow X_{j}(j=0,1)$ with

$$
x=x_{0}(t)+x_{1}(t) \text { and } \frac{t^{j}\left\|x_{j}(t)\right\|_{j}}{\rho(t)} \in L^{p_{j}}\left(\mathbf{R}^{+}, d t / t\right) \quad(j=0,1)
$$

with the norm $\|x\|_{J_{\rho, p_{0}, p_{1}}}=\inf \left\{\|\| x_{0}(t)\left\|_{0} / \rho(t)\right\|_{\left.L^{p_{0}(d t / t)}\right)}+\|t\| x_{1}(t)\left\|_{1} / \rho(t)\right\|_{L^{p_{1}(d t / t)}}\right\}$ and let $J_{\rho, p_{0}, p_{1}}(\bar{X})$ be the space of all those $x \in \Sigma \bar{X}$ such that there exists a strongly measurable function $u: \mathrm{R}^{+} \rightarrow \Delta \bar{X}$ with

$$
x=\int_{0}^{\infty} u(t) d t / t \text { and } \frac{t^{j}\|u(t)\|_{j}}{\rho(t)} \in L^{p_{j}}\left(\mathrm{R}^{+}, d t / t\right) \quad(j=0,1)
$$

with the norm $\|x\|_{J_{\rho, p_{0}, p_{1}}}=\inf \left\{\max _{j=0,1}\left(\left\|t^{j}\right\| u(t)\left\|_{j} / \rho(t)\right\|_{L_{j}^{p_{j}}(d t / t)}\right)\right\}$. 
For $t>0$, we recall Peetre's $J$ - and $K$-functionals in the following sense

$$
\begin{array}{ll}
J(t, x ; \bar{X})=\|x\|_{0} \vee\left(t\|x\|_{1}\right) & \text { for } x \in \Delta \bar{X}, \\
K(t, x ; \bar{X})=\inf \left\{\left\|x_{0}\right\|_{0}+t\left\|x_{1}\right\|_{1} \mid x=x_{0}+x_{1}, x_{j} \in X_{j}\right\} & \text { for } x \in \Sigma \bar{X} .
\end{array}
$$

We now turn to Brudnyi-Krugljak's $K$ - and $J$-methods

Definition 1.2. Let $\Phi$ be a Banach lattice over $\mathrm{R}^{+}$such that $1 \wedge t \in \Phi$ and

$$
\int_{0}^{\infty} 1 \wedge(1 / t)|x(t)| \frac{d t}{t}<\infty \text { for all } x \in \Phi
$$

then we define

$$
K_{\Phi}(\bar{X})=\left\{x \in \Sigma \bar{X} \mid\|x\|_{K_{\Phi}}=\|K(t, x ; \bar{X})\|_{\Phi}<\infty\right\}
$$

and $J_{\Phi}(\bar{X})$ as the space of all those $x \in \Sigma \bar{X}$ which permits a canonical representation $x=\int_{0}^{\infty} u(t) d t / t$ for a strongly measurable function $u: \mathrm{R}^{+} \rightarrow \Delta \bar{X}$ with the norm

$$
\|x\|_{J_{\Phi}}=\inf _{u} \| J\left(t, u(t) ; \bar{X} \|_{\Phi}<\infty\right.
$$

For the quasi-power function $\rho$, we define a continuous and increasing function $\varphi: \mathrm{R}^{+} \rightarrow \mathrm{R}^{+}$by

$$
\varphi^{-1}(t)=t^{1 / p_{0}} \rho\left(t^{-1 / q}\right)
$$

where $1 / q=1 / p_{0}-1 / p_{1}$. The assumption $\rho(1)$ gives $\varphi^{-1}(1)=\varphi(1)=1$. It is easy to see that $\lim _{t \rightarrow 0} \varphi^{-1}(t)=\lim _{t \rightarrow 0} \varphi(t)=0$ and $\lim _{t \rightarrow \infty} \varphi^{-1}(t)=\lim _{t \rightarrow \infty} \varphi(t)=\infty$. Moreover, $\varphi^{-1}$ is quasi-power satisfying

$$
\varphi^{-1}(\lambda t) \leq\left(\lambda^{1 / p_{\alpha}} \vee \lambda^{1 / p_{1-\alpha}}\right) \varphi^{-1}(t) \quad \forall \lambda>0, t>0,
$$

where $1 / p_{\alpha}=(1-\alpha) / p_{0}+\alpha / p_{1}$, and hence $\varphi$ is a Young's function. Let $\Phi$ be the weighted Orlicz space of all measurable functions

$$
f: \mathrm{R}^{+} \rightarrow \mathrm{C} \text { such that } \int_{0}^{\infty} \varphi\left(t^{-q / p_{0}}|f(t)|\right) t^{q} \frac{d t}{t}<\infty
$$

which is equipped with the Luxemberg norm 


$$
\|f\|_{\Phi}=\inf \left\{\lambda>0 \mid \int_{0}^{\infty} \varphi\left(t^{-q / p_{0}}|f(t)| / \lambda\right) t^{q} \frac{d t}{t} \leq 1\right\} .
$$

This kind of spaces was considered by Nilsson concerning interpolation spaces of the weighted $L^{p}$ spaces [11, Ex.5.3] in a more general sense. Observe

$$
\left(\lambda^{p_{a}} \wedge \lambda^{p_{1-\alpha}}\right) \varphi(t) \leq \varphi(\lambda t) \leq\left(\lambda^{p_{\alpha}} \vee \lambda^{p_{1-\alpha}}\right) \varphi(t) \quad \forall \lambda>0, t>0 .
$$

It follows that $1 \wedge t \in \Phi$ and $\int_{0}^{\infty} 1 \wedge(1 / t)|f(t)| \frac{d t}{t}<\infty$ for all $f \in \Phi$. Hence $K_{\Phi}$ and $J_{\Phi}$ are interpolation methods. Moreover, $\Phi$ satisfies the $\Delta_{2}$ condition since

$$
\varphi(2 t) \leq c^{p_{\alpha} \vee p_{1-\alpha}} \varphi(t) \quad \forall t>0 .
$$

The relationship between these interpolation methods is summarized in our main result as follows

THEOREM 1.1. Assume that $1 \leq p_{0}, p_{1} \leq \infty$ with $p_{0} \neq p_{1}, \rho$ is a quasi-power function and $\Phi$ is the weighted Orlicz space given by (1.4). For a Banach couple $\bar{X}$, we have

$$
K_{\Phi}(\bar{X})=K_{\rho, p_{0}, p_{1}}(\bar{X})=J_{\rho, p_{0}, p_{1}}(\bar{X})=J_{\Phi}(\bar{X})
$$

with norm estimates

$$
\begin{aligned}
& \|x\|_{K_{\Phi}} \leq 2\|x\|_{K_{\rho, p_{0}, p_{1}}},\|x\|_{K_{\rho, p_{0}, p_{1}}} \leq \frac{2}{\alpha \wedge(1-\alpha)}\|x\|_{J_{\rho, p_{0}, p_{1}}}, \\
& \|x\|_{J_{\rho, p_{0}, p_{1}}} \leq 4\|x\|_{J_{\Phi}}, \text { and }\|x\|_{J_{\Phi}} \leq \frac{e}{2}\|x\|_{K_{\Phi}}
\end{aligned}
$$

REMARKS. (i) If $p_{0}=p_{1}=p$, then we can also define $K_{\rho, p, p}(\bar{X})$ and $J_{\rho, p, p}(\bar{X})$ in the same way as in Def.1.1, but the space $\Phi$ does not make sense at this time. Alternatively, we set $L_{\rho}^{p}=\left\{f \mid f / \rho \in L^{p}\left(\mathrm{R}^{+}, d t / t\right)\right\}, K_{\rho}^{p}=K_{L_{\rho}^{p}}$ and $J_{\rho}^{p}=J_{L_{\rho}^{p}}$, and we can easily verify that

$$
K_{\rho, p, p}(\bar{X})=J_{\rho, p, p}(\bar{X})=J_{\rho}^{p}(\bar{X})=K_{\rho}^{p}(\bar{X}) .
$$

(ii) For $0 \leq \theta \leq 1$, let $\rho_{\theta}(t)=t^{\theta}$ be the power function, and let $L_{\theta}^{p}=L_{\rho_{\theta}}^{p}, K_{\theta}^{p}=K_{L_{\theta}^{p}}$ and $J_{\theta}^{p}=J_{L_{\theta}^{p}}$. Then $\varphi_{\theta}^{-1}(t)=t^{1 / p_{0}} \rho_{\theta}\left(t^{1 / q}\right)=t^{1 / p_{\theta}}$ and $\Phi=L_{\theta}^{p_{\theta}}$. It means that Theorem 1.1 together with (i) above can be viewed as an extension to the classical result

$$
K_{\rho_{\theta}, p_{0}, p_{1}}(\bar{X})=J_{\rho_{\theta}, p_{0}, p_{1}}(\bar{X})=J_{\theta}^{\rho_{\theta}}(\bar{X})=K_{\theta}^{\rho_{\theta}}(\bar{X}),
$$

for $0<\theta<1$ [1, Th.3.12.1]. 


\section{Equivalence $K_{\rho, p_{0}, p_{1}}(\bar{X})=J_{\rho, p_{0}, p_{1}}(\bar{X})$ and inclusion $K_{\Phi}(\bar{X}) \subseteq J_{\Phi}(\bar{X})$}

The proof of two lemmas in this section can be obtained by a modification of the statements for the classical case [1, Th.3.12.1\& Th.3.3.1], where $\rho_{\theta}$ is replaced by the quasi-power function $\rho$.

Lemma 2.1. $K_{\rho, p_{0}, p_{1}}(\bar{X})=J_{\rho, p_{0}, p_{1}}(\bar{X})$ with $\|x\|_{K_{\rho, p_{0}, p_{1}}} \leq \frac{2}{\alpha \wedge(1-\alpha)}\|x\|_{J_{\rho, p_{0}, p_{1}}}$.

Proof. For the inclusion $J_{\rho, p_{0}, p_{1}}(\bar{X}) \subseteq K_{\rho, p_{0}, p_{1}}(\bar{X})$, let $x=\int_{0}^{\infty} u(t) d t / t \in$
$J_{\rho, p_{0}, p_{1}}(\bar{X})$ and set

$$
x_{0}(t)=\int_{0}^{1} u(t \tau) d \tau / \tau \text { and } x_{1}(t)=\int_{1}^{\infty} u(t \tau) d \tau / \tau
$$

Then $x_{j}(t) \in X_{j}(j=0,1)$. According to Minkowski's inequality and (1.1), we have

$$
\begin{aligned}
\left\|\frac{\left\|x_{0}(t)\right\|_{0}}{\rho(t)}\right\|_{L^{p_{0}(d t / t)}} & \leq \int_{0}^{1}\left(\int_{0}^{\infty}\left(\frac{\|u(t \tau)\|_{0}}{\rho(t)}\right)^{p_{0}} \frac{d t}{t}\right)^{1 / p_{0}} \frac{d \tau}{\tau} \\
& \leq \frac{1}{\alpha \wedge(1-\alpha)}\left\|\frac{\|u(t)\|_{0}}{\rho(t)}\right\|_{L^{p_{0}(d t / t)}}
\end{aligned}
$$

and similarly

$$
\left\|\frac{t\left\|x_{1}(t)\right\|_{1}}{\rho(t)}\right\|_{L^{p_{1}(d t / t)}} \leq \frac{1}{\alpha \wedge(1-\alpha)}\left\|\frac{t\|u(t)\|_{1}}{\rho(t)}\right\|_{L^{p_{1}(d t / t)}}
$$

with the usual change if $p_{0}$ or $p_{1}=\infty$. That is, $x \in K_{\rho, p_{0}, p_{1}}(\bar{X})$ with

$$
\|x\|_{K_{\rho, p_{0}, p_{1}}} \leq \frac{2}{\alpha \wedge(1-\alpha)}\|x\|_{J_{\rho, p_{0}, p_{1}}} .
$$

The inversed inclusion $K_{\rho, p_{0}, p_{1}}(\bar{X}) \subseteq J_{\rho, p_{0}, p_{1}}(\bar{X})$ also relies upon Minkowski's inequality and (1.1). We leave the proof to the reader.

Lемма 2.2. $K_{\Phi}(\bar{X}) \subseteq J_{\Phi}(\bar{X})$ with $\|x\|_{J_{\Phi}} \leq \frac{e}{2}\|x\|_{K_{\Phi}}$.

Proof. Let $x \in K_{\Phi}(\bar{X})$. We set $K(t)=K(t, x ; \bar{X})$ for $t>0$ and claim that

$$
K(t)=O\left(t^{\alpha} \vee t^{1-\alpha}\right) .
$$

In fact, $(1 \wedge(s / t)) K(t) \leq K(s)$ for $t, s>0$ by [2, Lemma 3.1.1]. It follows that 


$$
\int_{0}^{\infty} \varphi\left(s^{-q / p_{0}}(1 \wedge(s / t)) K(t)\right) s^{q} \frac{d s}{s} \leq \int_{0}^{\infty} \varphi\left(s^{-q / p_{0}} K(s)\right) s^{q} \frac{d s}{s}
$$

while

$$
\begin{aligned}
& \int_{0}^{\infty} \varphi\left(s^{-q / p_{0}}(1 \wedge(s / t)) K(t)\right) s^{q} \frac{d s}{s} \\
& \geq\left(t^{-\alpha} K(t)\right)^{p_{\alpha}} \wedge\left(t^{\alpha-1} K(t)\right)^{p_{1-\alpha}} \int_{0}^{\infty} \varphi\left(s^{-q / p_{0}}(1 \wedge s)\right) s^{q} \frac{d s}{s}
\end{aligned}
$$

and

$$
\begin{aligned}
& \int_{0}^{\infty} \varphi\left(s^{-q / p_{0}}(1 \wedge s)\right) s^{q} \frac{d s}{s} \\
& \geq\left(\int_{0}^{1}\left(s^{(1-\alpha) p_{\alpha}} \wedge s^{\alpha p_{1-\alpha}}\right) \frac{d s}{s}+\int_{0}^{\infty}\left(s^{-\alpha p_{\alpha}} \wedge s^{-(1-\alpha) p_{1-\alpha}}\right) \frac{d s}{s}\right)>0
\end{aligned}
$$

by (1.5). Thus (2.1) is obtained since $K(s), 1 \wedge s \in \Phi$. Consequently, $(1 \wedge(1 / t)) K(t) \rightarrow 0$ as $t \rightarrow 0$ or $\infty$ by (2.1). According to the continuous version of the fundamental lemma [9], there is a canonical representation $x=\int_{0}^{\infty} u(t) d t / t$ for $\varepsilon>0$ such that

$$
J(t, u(t) ; \bar{X}) \leq \frac{e}{2}(1+\varepsilon) K(t, x ; \bar{X}) .
$$

This gives that $x \in J_{\Phi}(\bar{X})$ with $\|x\|_{J_{\Phi}} \leq \frac{e}{2}\|x\|_{K_{\Phi}}$.

\section{Duality relations}

The following duality argument plays an important role in the proof of Theorem 1.1. Let $\rho^{*}(t)=1 / \rho(1 / t)$ be the involution to $\rho$ and let $\psi: \mathrm{R}^{+} \rightarrow \mathrm{R}^{+}$ be the function satisfying

$$
\psi^{-1}(t)=t^{1 / p_{0}^{\prime}} \rho^{*}\left(t^{1 / q}\right)=t^{1 / p_{0}^{\prime}} / \rho\left(t^{-1 / q}\right)
$$

Then $\rho^{*}$ and $\psi^{-1}$ are also quasi-power functions such that

$$
\rho^{*}(\lambda t) \leq\left(\lambda^{\alpha} \vee \lambda^{1-\alpha}\right) \rho(t)
$$

and hence $\psi^{-1}(\lambda t) \leq\left(\lambda^{1 / p_{\alpha}^{\prime}} \vee \lambda^{1 / p_{1-\alpha}^{\prime}}\right) \psi^{-1}(t), \psi(\lambda t) \leq\left(\lambda^{p_{\alpha}^{\prime}} \wedge \lambda^{p_{1-\alpha}^{\prime}}\right) \psi(t)$ for all $\lambda>0, t>0$. Further we define $\Psi$ to be the corresponding weighted Orlicz space of all measurable functions 
INTERPOLATION METHODS OF CONSTANTS AND MEANS WITH QUASI-POWER 85

$$
f: \mathrm{R}^{+} \rightarrow \mathrm{C} \text { such that } \int_{0}^{\infty} \psi\left(t^{q / p_{0}^{\prime}}|f(t)|\right) t^{-q} \frac{d t}{t}<\infty
$$

with the Luxemberg norm.

Lemma 3.1. (i) $J_{\Phi}(\bar{X})^{\prime}=K_{\Psi}\left(\bar{X}^{\prime}\right)$ with $\left\|x^{\prime}\right\|_{J_{\Phi}(\bar{X})^{\prime}} \leq\left\|x^{\prime}\right\|_{K_{\Psi}\left(\bar{X}^{\prime}\right)}$; (ii) $J_{\Psi^{\prime}}(\bar{X})^{\prime}=$ $K_{\Phi}\left(\bar{X}^{\prime}\right)$.

Proof. Let $G_{\rho}$ be the \pm methods with respect to $\rho$ given by GustavssonPeetre [6]. Then $\Phi=G_{\rho}\left(L_{0}^{p_{0}}, L_{1}^{p_{1}}\right)$ such that $L_{0}^{p_{0}} \cap L_{1}^{p_{1}}$ is dense in $\Phi$, and $\Psi=G_{\rho^{*}}\left(L_{0}^{p_{0}^{\prime}}, L_{1}^{p_{1}^{\prime}}\right)$ by [11, Ex.5.3]. It follows that $\Psi=G_{\rho}\left(L_{0}^{p_{0}}, L_{1}^{p_{1}}\right)^{\prime}=\Phi^{\prime}$ by [11, Lemma 1.6]. According to [2, Th.3.7.2], we obtain

$$
J_{\Phi}(\bar{X})^{\prime}=K_{\Psi}\left(\bar{X}^{\prime}\right) \text { and } J_{\Psi}(\bar{X})^{\prime}=K_{\Phi}\left(\bar{X}^{\prime}\right) .
$$

For the inequality $\left\|x^{\prime}\right\|_{J_{\Phi}(\bar{X})^{\prime}} \leq\left\|x^{\prime}\right\|_{K_{\Psi}\left(\bar{X}^{\prime}\right)}$, it is enough to show that $(D \varphi)^{-1}(t) \leq D \psi(t)$ for all $t>0$, where $D \varphi$ means the derivative of $\varphi$, since $\left\|x^{\prime}\right\|_{J_{\Phi}(\bar{X})^{\prime}}=\left\|x^{\prime}\right\|_{K_{\Phi^{\prime}}\left(\bar{X}^{\prime}\right)}$ by [2, Th.3.7.2 \& Rmk.3.7.3]. In fact, the concavity of $\rho$ gives that $0 \leq D \rho(t) \leq \frac{\rho(t)}{t}$ and hence

$$
0 \leq \frac{1}{p_{0} \vee p_{1}} \leq \frac{1}{p_{0}}-\frac{t D \rho(t)}{q \rho(t)} \leq \frac{1}{p_{0} \wedge p_{1}} \leq 1 .
$$

By differentiating the identity $\varphi\left(t^{1 / p_{0}} \rho\left(t^{-1 / q}\right)\right)=t$, we have

$$
D \varphi\left(t^{1 / p_{0}} \rho\left(t^{-1 / q}\right)\right) t^{1 / p_{0}} \rho\left(t^{-1 / q}\right)\left(\frac{1}{p_{0}}-\frac{t^{-1 / q} D \rho\left(t^{-1 / q}\right)}{q \rho\left(t^{1 / q}\right)}\right)=t .
$$

Since $\varphi^{-1}(t)=t^{1 / p_{0}} \rho\left(t^{-1 / q}\right)$ and $\psi^{-1}(t)=t^{1 / p_{0}^{\prime}} / \rho\left(t^{-1 / q}\right)$, it implies that

$$
\psi^{-1}(t)=D \varphi\left(t^{1 / p_{0}} \rho\left(t^{-1 / q}\right)\right)\left(\frac{1}{p_{0}}-\frac{t D \rho(t)}{q \rho(t)}\right)
$$

and hence $\psi^{-1}(t) \leq D \varphi \circ \varphi^{-1}(t)$ as well as $\varphi^{-1}(t) \leq D \psi \circ \psi^{-1}(t)$. The inequality $(D \varphi)^{-1}(t) \leq D \psi(t)$ is obtained by the straightforward calculation.

For the $J_{\rho, p_{0}, p_{1}}$ and $K_{\rho, p_{0}, p_{1}}$ methods, we have

LEMMA 3.2

(i) $J_{\rho, p_{0}, p_{1}}(\bar{X})^{\prime}=K_{\rho^{*}, p_{0}^{\prime}, p_{1}^{\prime}}\left(\bar{X}^{\prime}\right)$ with $\left\|x^{\prime}\right\|_{J_{\rho, p_{0}, p_{1}}(\bar{X})^{\prime}} \leq\left\|x^{\prime}\right\|_{K_{\rho^{*}, p_{0}^{\prime}, p_{1}}\left(\bar{X}^{\prime}\right)} \leq 2\left\|x^{\prime}\right\|_{J_{\rho, p_{0}, p_{1}}(\bar{X})^{\prime}}$;

(ii) $K_{\rho, p_{0}, p_{1}}(\bar{X})^{\prime}=J_{\rho^{*}, p_{0}^{\prime} p_{0}^{\prime}}\left(\bar{X}^{\prime}\right)$.

Proof. For $x=\int_{0}^{\infty} u(t) d t / t \in J_{\rho, p_{0}, p_{1}}(\bar{X})$ and $x^{\prime}=x_{0}^{\prime}(t)+x_{1}^{\prime}(t) \in K_{\rho^{*}, p_{0}^{\prime}, p_{1}^{\prime}\left(\bar{X}^{\prime}\right)}$ 


$$
\left\langle x^{\prime}, x\right\rangle=\int_{0}^{\infty}\left\langle\frac{x_{0}^{\prime}(1 / t)}{\rho^{*}(1 / t)}, \frac{u(t)}{\rho(t)}\right\rangle \frac{d t}{t}+\int_{0}^{\infty}\left\langle\frac{x_{1}^{\prime}(1 / t)}{\rho^{*}(1 / t)}, \frac{u(t)}{\rho(t)}\right\rangle \frac{d t}{t} .
$$

Then $\left|\left\langle x^{\prime}, x\right\rangle\right| \leq\left\|x^{\prime}\right\|_{K_{\rho^{*}, p_{0}^{\prime}, p_{1}^{\prime}}\left(\bar{X}^{\prime}\right)} \cdot\left\|x^{\prime}\right\|_{J_{\rho, p_{0}, p_{1}}(\bar{X})}$, which gives

$$
K_{\rho^{*}, p_{0}^{\prime}, p_{1}^{\prime}}\left(\bar{X}^{\prime}\right) \subseteq J_{\rho, p_{0}, p_{1}}(\bar{X})^{\prime} \text { with }\left\|x^{\prime}\right\|_{J_{\rho, p_{0}, p_{1}}(\bar{X})^{\prime}} \leq\left\|x^{\prime}\right\|_{K_{\rho^{*}, p_{0}^{\prime}, p_{1}^{\prime}}\left(\bar{X}^{\prime}\right)^{\prime}} .
$$

On the other hand, let $x^{\prime} \in \Sigma \bar{X}^{\prime}$ then

$$
K\left(t, x^{\prime} ; \bar{X}^{\prime}\right)=\sup _{x \in \Delta \bar{X}} \frac{\left|\left\langle x^{\prime}, x\right\rangle\right|}{J(1 / t, x ; \bar{X})} \quad \text { for all } t>0
$$

by [1, 3.7.(1)]. So $\forall \varepsilon>0$ we can find strongly measurable functions $u: \mathrm{R}^{+} \rightarrow \Delta \bar{X}$ and $x_{j}^{\prime}: \mathrm{R}^{+} \rightarrow \Sigma \bar{X}^{\prime}$ such that $x^{\prime}=x_{0}^{\prime}+x_{1}^{\prime}$ and

$$
t^{j}\left\|x_{j}^{\prime}(t)\right\|_{X_{j}^{\prime}} \leq(1+\varepsilon) \frac{\left\langle x^{\prime}, u(1 / t)\right\rangle}{J(1 / t, u(1 / t) ; \bar{X})} \quad(j=0,1) .
$$

For an arbitrary measurable function $f: \mathrm{R}^{+} \rightarrow \mathrm{R}^{+} \cup\{0\}$ with $f / \rho \in L^{p_{0}}(d t / t) \cap L^{p_{1}}(d t / t)$, we see that

$$
x=\int_{0}^{\infty} \frac{f(t) u(t)}{J(t, u(t) ; \bar{X})} \frac{d t}{t} \in J_{\rho, p_{0}, p_{1}}(\bar{X}),
$$

and hence

$$
\begin{aligned}
&\left|\int_{0}^{\infty} \frac{f(1 / t)}{\rho(1 / t)} \cdot \frac{t^{j}\left\|x_{j}^{\prime}(t)\right\|_{X_{j}^{\prime}}}{\rho^{*}(t)} \frac{d t}{t}\right|=\int_{0}^{\infty} f(1 / t)\left\|x_{j}^{\prime}(t) t^{j}\right\|_{X_{j}^{\prime}} \frac{d t}{t} \\
& \leq(1+\varepsilon) \int_{0}^{\infty} \frac{f(1 / t)\left\langle x^{\prime}, u(1 / t)\right\rangle}{J(1 / t, u(1 / t) ; \bar{X})} \frac{d t}{t} \\
& \leq(1+\varepsilon)\left|\left\langle x^{\prime}, x\right\rangle\right| \leq(1+\varepsilon)\left\|x^{\prime}\right\|_{J_{\rho, p_{0}, p_{1}}^{\prime}(\bar{X})^{\prime}}^{\prime}\|x\|_{J_{\rho, p_{0}, p_{1}}(\bar{X})}
\end{aligned}
$$

for $j=0,1$. This follows that $x^{\prime} \in K_{\rho^{*}, p_{0}^{\prime}, p_{1}^{\prime}}\left(\bar{X}^{\prime}\right)$ with $\|x\|_{K_{\rho^{*}, p_{0}^{\prime}, p_{1}^{\prime}}\left(\bar{X}^{\prime}\right)} \leq 2\left\|x^{\prime}\right\|_{J_{\rho, p_{0}, p_{1}}(\bar{X})^{\prime}} \cdot$

The other equivalence $K_{\rho, p_{0}, p_{1}}(\bar{X})^{\prime}=J_{\rho^{*}, p_{0}^{\prime}, p_{1}^{\prime}}\left(\bar{X}^{\prime}\right)$ can be obtained by the equivalence in Lemma 2.1.

\section{Equivalence $J_{\rho, p_{0}, p_{1}}(\bar{X})=J_{\Phi}(\bar{X})$}

For $t>0$ and $x \in \Sigma \bar{X}$ recall that the $E$-functional is defined by

$$
E(t, x ; \bar{X})=\inf \left\{\left\|x-x_{0}\right\|_{1} \mid x_{0} \in X_{0} \text { with }\left\|x_{0}\right\|_{0} \leq t\right\} .
$$


It is known from [2, Sec.3.1] that $E(t, x ; \bar{X})$ is a nonincreasing function with

$$
\lim _{t \rightarrow 0} E(t, x ; \bar{X})=\infty \text { and } \lim _{t \rightarrow \infty} E(t, x ; \bar{X})=0 .
$$

Furthermore,

$$
E(t, x ; \bar{X})=\sup _{s>0} \frac{K(s, x ; \bar{X})-t}{s} .
$$

For $x \in K_{\rho, p_{0}, p_{1}}(\bar{X})$, its norm can be given by using the $E$-functional in the following way

$$
\begin{aligned}
\|x\|_{K_{\rho, p_{0}, p_{1}}}= & \inf \left\{\|w(t) / \rho(t)\|_{L^{p_{0}}(d t / t)}+\|t E(w(t), x ; \bar{X}) / \rho(t)\|_{L^{p_{1}}(d t / t)} \mid\right. \\
& \left.w: \mathrm{R}^{+} \rightarrow \mathrm{R}^{+} \cup\{0\} \text { is measurable }\right\} .
\end{aligned}
$$

The proof of the following lemma involves some meticulous calculations by developing a technique for the classical case when $\rho=\rho_{\theta}$ due to Holmstedt [8, Sec.5].

LEMma 4.1. $K_{\rho, p_{0}, p_{1}}(\bar{X})^{0} \subseteq K_{\Phi}(\bar{X})$ with $\|x\|_{K_{\Phi}} \leq 2\|x\|_{K_{\rho, p_{0}, p_{1}}}$.

Proof. Let $x \in \Delta \bar{X}$ with $x \neq 0$ and let $E(t)=E(t, x ; \bar{X})$. For a measurable function $w \geq 0$ defined in $\mathrm{R}^{+}$, we set

$$
I_{0}(w)=\|w(t) / \rho(t)\|_{L^{p_{0}(d t / t)}} \text { and } I_{1}(w)=\|t E(w(t)) / \rho(t)\|_{L^{p_{1}(d t / t)}} .
$$

For $\varphi$ given by (1.2) and $\lambda=\|x\|_{K_{\Phi}}^{p_{1}-p_{0}}$, we define a function $v^{-1}$ in $\mathrm{R}^{+}$in the form

$$
v^{-1}(s)=\varphi\left(\lambda^{1 /\left(p_{0}-p_{1}\right)} s^{-q / p_{1}} E(s)^{q / p_{0}}\right)^{-1 / q} .
$$

Then $v^{-1}$ is continuous and increasing with $\lim _{s \rightarrow 0} v^{-1}(s)=0$ and $\lim _{s \rightarrow \infty} v^{-1}(s)=\infty$. Thus $v^{-1}$ has an inverse function, which is denoted by $v$, with the same properties. Now (4.4) is equivalent to

$$
t=\varphi\left(\lambda^{1 /\left(p_{0}-p_{1}\right)} v(t)^{-q / p_{1}} E(v(t))^{q / p_{0}}\right)^{1 / q}
$$

and hence

$$
\lambda\left(\frac{v(t)}{\rho(t)}\right)^{p_{0}}=\left(\frac{t E(v(t))}{\rho(t)}\right)^{p_{1}} .
$$

Assume that $w: \mathrm{R}^{+} \rightarrow \mathrm{R}^{+} \cup\{0\}$ is measurable with $I_{0}(w)+I_{1}(w)<\infty$. Then we have

$$
I_{0}(v)+I_{1}(v) \leq\left(I_{0}(w)+\lambda^{1 / p_{0}} I_{1}(w)^{p_{1} / p_{0}}+\lambda^{1 / p_{1}} I_{0}(w)^{p_{0} / p_{1}}+I_{1}(w)\right)<\infty .
$$


It implies that $v(t) / \rho(t) \rightarrow 0$ as $t \rightarrow 0$ or $\infty$, and hence

$$
\left(\frac{v(t)}{t E(v(t))}\right)^{q}=\lambda^{-q / p_{1}}\left(\frac{v(t)}{\rho(t)}\right)^{q-p_{0} / p_{1}}=\lambda^{-q / p_{1}}\left(\frac{v(t)}{\rho(t)}\right)^{p_{0}} \rightarrow 0 \quad \text { as } t \rightarrow 0 \text { or } \infty .
$$

Consequently, we obtain

$s^{q} E(s)^{-q} \varphi\left(\lambda^{1 /\left(p_{0}-p_{1}\right)} s^{-q / p_{1}} E(s)^{q / p_{0}}\right)^{-1 / q}=v^{-1}(s)^{-q}\left(\frac{s}{E(s)}\right)^{q} \rightarrow 0 \quad$ as $s \rightarrow 0$ or $\infty$.

With a changing of variables by putting $t=v^{-1}(s)$ and with an integration by parts we get

$$
\begin{aligned}
I_{0}(v)^{p_{0}} & =\int_{0}^{\infty}\left(\frac{v(t)}{\rho(t)}\right)^{p_{0}} \frac{d t}{t}=\int_{0}^{\infty}\left(\frac{s}{\rho\left(v^{-1}(s)\right)}\right)^{p_{0}} \frac{d v^{-1}(s)}{v^{-1}(s)} \\
& =-\frac{1}{q} \int_{0}^{\infty}\left(\frac{s}{\varphi^{-1}\left(v^{-1}(s)^{-q}\right)}\right)^{p_{0}} d\left(v^{-1}(s)^{-q}\right) \\
& =-\frac{\lambda^{q / p_{1}}}{q} \int_{0}^{\infty} s^{q} E(s)^{-q} d \varphi\left(\lambda^{1 /\left(p_{0}-p_{1}\right)} s^{-q / p_{1}} E(s)^{q / p_{0}}\right) \\
& =\lambda^{q / p_{1}} \int_{0}^{\infty} \varphi\left(\lambda^{1 /\left(p_{0}-p_{1}\right)} s^{-q / p_{1}} E(s)^{q / p_{0}}\right)(s / E(s))^{q} \frac{d(s / E(s))}{s / E(s)}
\end{aligned}
$$

and similarly

$$
\begin{aligned}
I_{1}(v)^{p_{1}} & =\int_{0}^{\infty}\left(\frac{t E(v(t))}{\rho(t)}\right)^{p_{1}} \frac{d t}{t} \\
& =\lambda^{q / p_{0}} \int_{0}^{\infty} \varphi\left(\lambda^{1 /\left(p_{0}-p_{1}\right)} s^{-q / p_{1}} E(s)^{q / p_{0}}\right)(s / E(s))^{q} \frac{d(s / E(s))}{s / E(s)} .
\end{aligned}
$$

Let $t=s / E(s)$ and $K(t)=K(t, x ; \bar{X})$, we have

$$
s^{-q / p_{1}} E(s)^{q / p_{0}}=t^{-q / p_{0}} s=t^{-q / p_{0}} \cdot t E(s) \geq \frac{1}{2} t^{-q / p_{0}} K(t)
$$

by (4.2). It implies that

$$
I_{j}(v)^{p_{j}} \geq \frac{\lambda^{q / p_{1-j}}}{2^{p_{0} \vee p_{1}}} \int_{0}^{\infty} \varphi\left(\lambda^{1 /\left(p_{01}-p_{1}\right)} t^{-q / p_{0}} K(t)\right) t^{q} \frac{d t}{t} \geq \frac{\|x\|_{K_{\Phi}}^{p_{j}}}{2^{p_{0} \vee p_{1}}}
$$

for $j=0,1$. Subsequently, 


$$
\|x\|_{K_{\Phi}} \leq 2^{\left(p_{1-j} / p_{j}\right) \vee 1} I_{j}(v) \quad(j=0,1) .
$$

Then by (4.5-6) we have

$$
\begin{aligned}
& \|x\|_{K_{\Phi}} \leq 2^{\left(p_{0} / p_{1}\right) \vee\left(p_{1} / p_{0}\right)-1} \\
& \left(I_{0}(w)+\|x\|_{K_{\Phi}}^{1-p_{1} / p_{0}} I_{1}(w)^{p_{1} / p_{0}}+\|x\|_{K_{\Phi}}^{1-p_{0} / p_{1}} I_{0}(w)^{p_{0} / p_{1}}+I_{1}(w)\right) .
\end{aligned}
$$

It follows that $1 \leq 2\left(I_{0}(w) /\|x\|_{K_{\Phi}}+I_{1}(w) /\|x\|_{K_{\Phi}}\right)$. That is, $\|x\|_{K_{\Phi}} \leq 2$ $\left(I_{0}(w)+I_{1}(w)\right)$ holds for all measurable functions $w$. Therefore we have $\|x\|_{K_{\Phi}} \leq 2\|x\|_{K_{\rho, p_{0}, p_{1}}}$ by (4.3).

Now we are ready to complete the proof of Theorem 1.1: if $X$ is an intermediate space for $\bar{X}$ and $x \in X$, observe that

$$
\|x\|_{X}=\sup \left\{\mid\left\langle x^{\prime}, x\right\rangle \| x^{\prime} \in\left(X^{\prime}\right)^{0} \text { with }\left\|x^{\prime}\right\|_{X^{\prime}} \leq 1\right\} .
$$

In particular, for $x \in J_{\Phi}(\bar{X})$, we have

$$
\begin{aligned}
\|x\|_{J_{\rho, p_{0}, p_{1}}} & =\sup \left\{\mid\left\langle x^{\prime}, x\right\rangle \| x^{\prime} \in\left(J_{\rho, p_{0}, p_{1}}(\bar{X})^{\prime}\right)^{0} \text { with }\left\|x^{\prime}\right\|_{J_{\rho, p_{0}, p_{1}}(\bar{X})^{\prime}} \leq 1\right\} \\
& \left.\leq 2 \sup \left\{\mid\left\langle x^{\prime}, x\right\rangle \| x^{\prime} \in K_{\rho^{*}, p_{0}^{\prime}, p_{1}^{\prime}}\left(\bar{X}^{\prime}\right)^{0} \text { with }\left\|x^{\prime}\right\|_{K_{\rho^{*}, p_{0}^{\prime}, p_{1}^{\prime}}} \bar{X}^{\prime}\right) \leq 1\right\} \\
& \leq 4 \sup \left\{\mid\left\langle x^{\prime}, x\right\rangle \| x^{\prime} \in K_{\Psi}\left(\bar{X}^{\prime}\right) \text { with }\left\|x^{\prime}\right\|_{K_{K^{\prime}}\left(\bar{X}^{\prime}\right)} \leq 1\right\} \\
& \leq 4 \sup \left\{\mid\left\langle x^{\prime}, x\right\rangle \| x^{\prime} \in J_{\Phi}(\bar{X})^{\prime} \text { with }\left\|x^{\prime}\right\|_{J_{\Phi}(\bar{X})^{\prime}} \leq 1\right\}
\end{aligned}
$$

by (4.7), Lemma 3.2, Lemma 4.1 and Lemma 3.1. This gives that $J_{\Phi}(\bar{X}) \subseteq$ $J_{\rho, p_{0}, p_{1}}(\bar{X})$ with $\|x\|_{J_{\rho, p_{0}, p_{1}}} \leq 4\|x\|_{J_{\Phi}}$, and symmetrically $J_{\Psi}\left(\bar{X}^{\prime}\right) \subseteq J_{\rho^{*}, p_{0}^{\prime}, p_{1}^{\prime}}\left(\bar{X}^{\prime}\right)$. Since

$$
\begin{array}{ll}
J_{\rho^{*}, p_{0}^{\prime}, p_{1}^{\prime}}\left(\bar{X}^{\prime}\right)=K_{\rho^{*}, p_{0}^{\prime}, p_{1}^{\prime}}\left(\bar{X}^{\prime}\right)=J_{\rho, p_{0}, p_{1}}(\bar{X})^{\prime} & \text { (by Lemma } 2.1 \text { and Lemma 3.2) } \\
J_{\Phi}(\bar{X})^{\prime}=K_{\Psi}\left(\bar{X}^{\prime}\right) \subseteq J_{\Psi}\left(\bar{X}^{\prime}\right) & \text { (by Lemma 3.1 and Lemma 2.2), }
\end{array}
$$

it implies that $J_{\Phi}(\bar{X})^{\prime} \subseteq J_{\rho, p_{0}, p_{1}}(\bar{X})^{\prime}$. Therefore, we obtain the last equivalence in Theorem 1.1 as follows

$$
J_{\Phi}(\bar{X})=J_{\rho, p_{0}, p_{1}}(\bar{X}) .
$$

REMARKS. (i) From the above proof to the main theorem, one can see how the classical method with power parameters is naturally extended to the case with quasi-power parameters. As mentioned at the beginning of this paper, we could also follow the abstract formulas

$$
K_{\rho, p_{0}, p_{1}}(\bar{X})=K_{K_{\rho, p_{0}, p_{1}}\left(\bar{L}^{\infty}\right)}(\bar{X}) \text { and } J_{\rho, p_{0}, p_{1}}(\bar{X})=J_{J_{\rho, p_{0}, p_{1}}\left(\bar{L}^{1}\right)}(\bar{X}),
$$

where $\bar{L}^{p}=\left(L_{0}^{p}, L_{1}^{p}\right)$ for $p=1, \infty$, according to [2, Th.4.2.11 \& 4.2.33], and 
prove our main theorem by calculating $\Phi=K_{\rho, p_{0}, p_{1}}\left(\bar{L}^{\infty}\right)=J_{\rho, p_{0}, p_{1}}\left(\bar{L}^{1}\right)$ instead. As far as we can see, however, that such a calculation can not shorten the proof. We observe, for instance, that $K\left(t, f ; \bar{L}^{\infty}\right)=\hat{f}(t)$ by [2, (3.1.24)], where $\hat{f}$ is the least concave majorant for $f \in \bar{L}^{\infty}$. It implies that

$$
E\left(t, f ; \bar{L}^{\infty}\right)=\sup _{s>0} \frac{\hat{f}(s)-t}{s} \geq \frac{|f(s)|-t}{s} \text { for all } s, t>0
$$

by (4.2). Therefore, the proof of the inclusion $K_{\rho, p_{0}, p_{1}}\left(\bar{L}^{\infty}\right) \subseteq \Phi$ is quite similar to that of Lemma 4.1. The only change is to replace $K\left(t, f ; \bar{L}^{\infty}\right)$ by $|f(t)|$ and to set $\lambda=\|f\|_{\Phi}^{p_{1}-p_{0}}$.

(ii) For the equivalence $J_{\Phi}(\bar{X})=K_{\Phi}(\bar{X})$, it is also possible to apply [2, Cor.3.5.15] by verifying that $\Phi$ is a quasi-power parameter in the sense that the linear operator $S$ given by $(S f)(t)=\int_{0}^{\infty}(1 \wedge(t / s)) f(s) \frac{d s}{s}$ is bounded on $\Phi$. But this is not trivial since it involves the Hardy-type inequality for weighted Orlicz spaces. In addtion, our direct proof for $K_{\Phi}(\bar{X}) \subseteq J_{\Phi}(\bar{X})$ in Lemma 2.2 gives a much better norm estimate $\|x\|_{J_{\Phi}} \leq \frac{e}{2}\|x\|_{K_{\Phi}}$ than that in the general case $\|x\|_{J_{\Phi}} \leq 18\|x\|_{K_{\Phi}}$.

\section{Connection with complex interpolation}

Let

$$
\rho_{\theta, n}(t)=t^{\theta}\left(1+\frac{\theta(1-\theta)}{|n|}|\log t|\right)^{n} \text { for } t>0,
$$

where $0<\theta<1$ and $n=0, \pm 1, \pm 2, \ldots$. For $1 \leq p_{0}, p_{1} \leq \infty$ with $p_{0} \neq p_{1}$ let

$$
\varphi^{-1}(t)=t^{1 / p_{0}} \rho_{\theta, n}\left(t^{1 / p_{1}-1 / p_{0}}\right)
$$

and let $\Phi_{\theta, n}^{p_{0}, p_{1}}$ be the corresponding weighted Orlicz space given by (1.6), then Theorem 1.1 gives that

$$
J_{\rho \theta, n}, p_{0}, p_{1}(\bar{X})=K_{\rho_{\theta, n} ; p_{0}, p_{1}}(\bar{X})=K_{\Phi_{\theta, n}^{p_{0}, p_{1}}}(\bar{X})=J_{\Phi_{\theta, n}^{p_{0}, p_{1}}}(\bar{X})
$$

In this section, we investigate the relationship between the real interpolation methods given by (5.2) and the complex interpolation methods with derivatives for a Banach couple of Fourier type, as well as some related topics. For $1 \leq p \leq 2$, a Banach space $X$ is called of Fourier type $p$, if for any $f \in L^{p}(\mathrm{R}, d t ; X)$, the $X$-valued $L^{p}$-space, the Fourier transform $F f \in L^{p^{\prime}}(\mathrm{R}, d t ; X)$.

Now we consider the complex interpolation with derivatives for a Banach couple $\bar{X}$. for the strip $\mathrm{S}=\{z \in \mathrm{C} \mid 0 \leq \operatorname{Re} z \leq 1\}$, let $A^{b}(\mathrm{~S}, \bar{X})$ be the Banach 
space of all boundedly continuous functions $f: S \rightarrow \Sigma \bar{X}$ such that $f$ is analytic in int $\mathrm{S}$ and $f(j+i t) \in X_{j}$ for $t \in \mathrm{R}(j=0,1)$ with the norm $\|f\|_{\infty}=\max _{j=0,1}\left\{\sup _{t \in \mathrm{R}}\|f(j+i t)\|\right\}$. The complex interpolation spaces with $n$th derivative at $\theta$, where $n \geq 0$, is defined in the following way:

$$
C_{\theta(n)}(\bar{X})=\left\{x \in \Sigma \bar{X} \mid x=\frac{1}{n !} f^{(n)}(\theta) \text { for some } f \in A^{b}(\mathrm{~S}, \bar{X})\right\}
$$

with the norm $\|x\|_{C_{\theta(n)}}=\inf \left\{\|f\|_{\infty} \mid x=\frac{1}{n !} f^{(n)}(\theta)\right\}$ and

$$
\begin{gathered}
C_{\theta(-n)}(\bar{X})=\left\{x \in \Sigma \bar{X} \mid x=f(\theta) \text { for some } f \in A^{b}(\mathrm{~S}, \bar{X})\right. \text { but } \\
\left.f^{\prime}(\theta)=f^{\prime \prime}(\theta)=\cdots=f^{(n)}(\theta)=0\right\}
\end{gathered}
$$

with the norm $\|x\|_{C_{\theta(-n)}}=\inf \left\{\|f\|_{\infty} \mid x=f(\theta)\right\}$. For notational convenience, we set $C_{\theta}(\bar{X})=C_{\theta(0)}(\bar{X})$ and let $K_{\theta}^{p}(\bar{X})$ be the classical real interpolation space with the parameter $L_{\theta}^{p}$. Peetre found that if $X_{j}$ is of Fourier type $p_{j}$ with $1 \leq p_{j} \leq 2(j=0,1)$, then

$$
K_{\theta}^{p}(\bar{X}) \subseteq C_{\theta}(\bar{X}) \subseteq K_{\theta}^{p^{\prime}}(\bar{X}),
$$

where $1 / p=(1-\theta) / p_{0}+\theta / p_{1}[12]$. Carro, Cerdà and Soria used a variant of the $K_{\rho_{\theta, n}, p_{0}, p_{1}}$-methods and generalized this result to the $C_{\theta(n)}$-methods for $n>0$, i.e.

$$
K_{\rho_{\theta, n}, p_{0}, p_{1}}(\bar{X}) \subseteq C_{\theta(n)}(\bar{X}) \subseteq K_{\rho_{\theta, n}, p_{0}^{\prime}, p_{1}^{\prime}}(\bar{X})
$$

[3, Prop.6].

Now we will give a more natural description for this inculsion to the $C_{\theta(n)^{-}}$ methods for all $n$ and even prove that the inclusion is optimal. Our proof involves (5.3) and the following reiteration theorem

Lemma 5.1. ([5, Rmk.5.8(ii)] \& [4, Th.5.4(ii)].

For $0<\theta_{0}, \theta_{1}, \eta<1$ with $\theta_{0} \neq \theta_{1}, 1 \leq p_{0}, p_{1} \leq \infty$ with $p_{0} \neq p_{1}, n=0$, $\pm 1, \pm 2, \ldots$, let

$$
\theta=(1-\eta) \theta_{0}+\eta \theta_{1}, 1 / q=1 / p_{0}-1 / p_{1} \text { and } \varphi^{-1}(t)=t^{1 / p_{0}} \rho_{\theta, n}\left(t^{-1 / q}\right) .
$$

Then

(i) $C_{\theta(n)}(\bar{X})=C_{\eta(n)}\left(C_{\theta_{0}}(\bar{X}), C_{\theta_{1}}(\bar{X})\right)$;

(ii) $K_{\Phi}(\bar{X})=C_{\eta(n)}\left(K_{\theta_{0}}^{p_{0}}(\bar{X}), K_{\theta_{1}}^{p_{1}}(\bar{X})\right)$, where $\Phi$ is the weighted Orlicz space given by

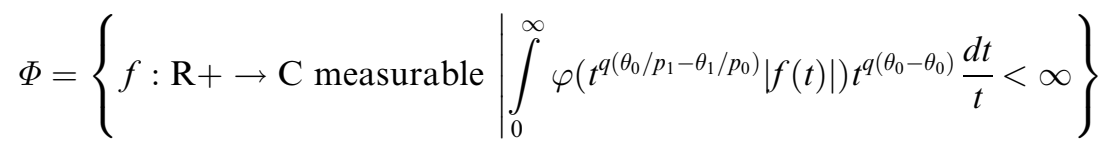


with the corresponding Luxemberg norm.

Observe that $\Phi_{\theta, n}^{p_{0}, p_{1}}=\Phi$ in Lemma 5.1 with $\theta_{0}=0, \theta_{1}=1$. Here is our result

Theorem 5.1. Let $1 \leq p_{0}, p_{1} \leq 2$ with $p_{0} \neq p_{1}, n=0, \pm 1, \pm 2, \ldots$, and let $\bar{X}$ be a Banach couple. If $X_{j}$ is of Fourier type $p_{j}(j=0,1)$, then

$$
K_{\Phi_{\theta, n}^{p_{0}, p_{1}}}(\bar{X}) \subseteq C_{\theta(n)}(\bar{X}) \subseteq K_{\Phi_{\theta, n}^{p_{0}^{\prime}, p_{1}^{\prime}}}(\bar{X}) .
$$

Moreover, the inclusion cannot be improved in the sense that the Banach lattice $\Phi_{\theta, n}^{p_{0}, p_{1}}$ (resp. $\left.\Phi_{\theta, n}^{p_{0}^{\prime}, p_{1}^{\prime}}\right)$ cannot, in general, be replaced with another Banach lattice with $\Phi_{\theta, n}^{p_{0}, p_{1}}$ (resp. $\left.\Phi_{\theta, n}^{p_{0}^{\prime}, p_{1}^{\prime}}\right)$ as a sublattice (resp. superlattice).

Proof. We choose $\theta_{0}, \eta$ such that $0<\theta_{0}<\theta<1-\theta_{0}=\theta_{1}<1$ and $\theta=(1-\eta) \theta_{0}+\eta \theta_{1}$. Let $1 / q=1 / p_{0}-1 / p_{1}, 1 / r_{j}=\left(1-\theta_{j}\right) / p_{0}+\theta_{j} / p_{1}(j=0,1)$ and $1 / r=1 / r_{0}-1 / r_{1}=\left(1-2 \theta_{0}\right) / q$. So $K_{\theta_{j}}^{r_{j}}(\bar{X}) \subseteq C_{\theta_{j}}(\bar{X}) \subseteq K_{\theta_{j}}^{r_{j}^{\prime}}(\bar{X})(j=0,1)$ by (5.3) and hence

$$
C_{\eta(n)}\left(K_{\theta_{0}}^{r_{0}}(\bar{X}), K_{\theta_{1}}^{r_{1}}(\bar{X})\right) \subseteq C_{\eta(n)}\left(C_{\theta_{0}}(\bar{X}), C_{\theta_{1}}(\bar{X})\right) \subseteq C_{\eta(n)}\left(K_{\theta_{0}}^{r_{0}^{\prime}}(\bar{X}), K_{\theta_{1}}^{r_{1}^{\prime}}(\bar{X})\right) .
$$

Observe $C_{\eta(n)}\left(C_{\theta_{0}}(\bar{X}), C_{\theta_{1}}(\bar{X})\right)=C_{\theta(n)}(\bar{X})$ by Lemma 5.1(i). Furthermore, the calculation $r\left(\theta_{1}-\theta_{0}\right)=q$ and $r\left(\theta_{0} / r_{1}-\theta_{1} / r_{0}\right)=-q / p_{0}$, together with Lemma 5.1(ii), gives that

$$
C_{\eta(n)}\left(K_{\theta_{0}}^{r_{0}}(\bar{X}), K_{\theta_{1}}^{r_{1}}(\bar{X})\right)=K_{\Phi_{\theta, n}^{p_{0}, p_{1}}}(\bar{X}) \text { and } C_{\eta(n)}\left(K_{\theta_{0}}^{r_{0}^{\prime}}(\bar{X}), K_{\theta_{1}}^{r_{1}^{\prime}}(\bar{X})\right)=K_{\Phi_{\theta, n}^{p_{0}^{\prime}, p_{1}^{\prime}}}(\bar{X}) .
$$

Thus the inclusion (5.5) is immediate.

If we put $\bar{L}^{p_{0}, p_{1}}=\left(\bar{L}_{0}^{p_{0}}, \bar{L}_{1}^{p_{1}}\right)$, then $L_{j}^{p_{j}}$ is of Fourier type $p_{j}$ and $\Phi_{\theta, n}^{p_{0}, p_{1}}=C_{\theta(n)}\left(\bar{L}^{p_{0}, p_{1}}\right)$ by [4. Ex.5.3]. In particular, $K_{\theta}^{p}\left(\bar{L}^{p_{0}, p_{1}}\right)=C_{\theta}\left(\bar{L}^{p_{0}, p_{1}}\right)=L_{\theta}^{p}$ by $[1, T h .5 .5 .1 \& 5.5 .3]$. It implies that

$$
K_{\Phi_{\theta, n}^{p_{0}, p_{1}}}\left(\bar{L}^{p_{0}, p_{1}}\right)=C_{\eta(n)}\left(K_{\theta_{0}}^{r_{0}}\left(\bar{L}^{p_{0}, p_{1}}\right), K_{\theta_{1}}^{r_{1}}\left(\bar{L}^{p_{0}, p_{1}}\right)\right)=C_{\eta(n)}\left(C_{\theta_{0}}\left(\bar{L}^{p_{0}, p_{1}}\right), C_{\theta_{1}}\left(\bar{L}^{p_{0}, p_{1}}\right)\right),
$$

and hence

$$
K_{\Phi_{\theta, n}^{p_{0}, p_{1}}}\left(\bar{L}^{p_{0}, p_{1}}\right)=C_{\theta(n)}\left(\bar{L}^{p_{0}, p_{1}}\right)=\Phi_{\theta, n}^{p_{0}, p_{1}} .
$$

Similarly, let $\bar{L}^{p_{0}^{\prime}, p_{1}^{\prime}}=\left(L_{0}^{p_{0}^{\prime}}, L_{1}^{p_{1}^{\prime}}\right)$, then $L_{j}^{p_{j}^{\prime}}=\left(L_{j}^{p_{j}}\right)^{\prime}$ is also of Fourier type $p_{j}$ and

$$
K_{\Phi_{\theta, n}^{p_{0}^{\prime}, p_{1}^{\prime}}}\left(\bar{L}^{p_{0}^{\prime}, p_{1}^{\prime}}\right)=C_{\theta(n)}\left(\bar{L}^{p_{0}^{\prime}, p_{1}^{\prime}}\right)=\Phi_{\theta, n}^{p_{0}^{\prime}, p_{1}^{\prime}}
$$

That is, the inclusion (5.5) cannot be improved. 
REMARKs. (i) Inclusion (5.4) can also be reached by combining (5.5) and (5.2).

(ii) It is easy to prove that the identity (5.6) is valid for all $1 \leq p_{0}, p_{1} \leq \infty$, for instance, by using reiteration. Especially, we have

$$
K_{\Phi_{\theta, n}^{1, \infty}}\left(\bar{L}^{1, \infty}\right)=C_{\theta(n)}\left(\bar{L}^{1, \infty}\right)=\Phi_{\theta, n}^{1, \infty}
$$

\section{On non-commutative $L^{1}$-space}

In the rest of this paper, we extend the identity of the real and complex interpolation in (5.7) to the non-commutative case in the sense of HaagerupPisier [7]. For a Banach space $X$, we denote by $H_{\infty}(\mathrm{S}, X)$ the space of all bounded analytic functions $f: \mathrm{S} \rightarrow X$ equipped with norm $\|f\|_{\infty}=\sup _{z \in S}\|f\|_{X}$.

Each $f$ in $H_{\infty}(\mathrm{S}, X)$ has boundary values in the weak topology, which can be considered as bounded linear operators $T_{f, j}: L^{1}\left(\mathrm{R}_{j}\right) \rightarrow X$ where

$$
\mathrm{R}_{j}=\{z \in \mathrm{C} \mid \operatorname{Re} z=j\} \quad(j=0,1) .
$$

Then $X$ is said to have the analytic Radon-Nikodym property if every function in $H_{\infty}(\mathrm{S}, X)$ admits radial limits at almost every point in the boundary of the strip S. Now let $\bar{X}$ be a Banach couple and let $n=0, \pm 1, \pm 2, \ldots$ We define the interpolation space $C^{\theta(n)}(\bar{X})$ by replacing $A^{b}(\mathrm{~S}, \bar{X})$ in the definition of the interpolation space $C_{\theta(n)}(\bar{X})$ with the following space of analytic functions on $\mathrm{S}$

$$
H_{\infty}(\mathrm{S}, \bar{X})=\left\{f \in H_{\infty}(\mathrm{S}, \Sigma \bar{X}) \mid T_{f, j}: L^{1}\left(\mathrm{R}_{j}\right) \rightarrow X_{j} \text { is bounded }(j=0,1)\right\}
$$

with norm $\|f\|_{\infty}=\left\|T_{f, 0}\right\|_{0} \vee\left\|T_{f, 1}\right\|_{1}$. In general, we have the duality property

$$
C_{\theta(n)}(\bar{X})^{\prime}=C^{\theta(-n)}\left(\bar{X}^{\prime}\right),
$$

and in particular, if $X_{0} \subseteq X_{1}$ and if $X_{1}$ has the analytic Radon-Nikodym property, then the equivalence

$$
C_{\theta(n)}(\bar{X})=C^{\theta(n)}(\bar{X})
$$

holds. The last identity is a trivial extension of [7, Prop.3.1].

As stated in [7, Sec.3], we study a Banach space $X$ such that there are a linear injection $i: X^{\prime} \rightarrow X$ with dense range and norm 1 and an involution $x \rightarrow x^{\prime}$ on $X^{\prime}$ such that $\left\langle x^{\prime}, i(x)\right\rangle \geq 0 \forall x \in X^{\prime}$ and $\langle y, i(x)\rangle=\langle i(y), x\rangle \forall x, y \in X^{\prime}$. Thus $\left(X^{\prime}, X\right)$ can be viewed as a Banach couple by identifying $X^{\prime}$ with $i\left(X^{\prime}\right) \subseteq X$, and $X^{\prime}$ can be embedded into a Hilbert space $H$, which is the completion of the prehilbertian space $X^{\prime}$ equipped with the scalar product $\langle x \mid y\rangle=\langle y, i(x)\rangle \forall x, y \in X^{\prime}$. If in addition $X^{\prime \prime}$ has the analytic Radon-Nikodym property, we can obtain $H$ by the complex interpolation 


$$
C_{1 / 2}\left(X^{\prime}, X\right)=H
$$

with equal norms [HP, Prop.3.2]. A typical example of this kind of Banach spaces is the predual of a von Neumman algebra, which is called a noncommutative $L^{1}$-space [7]. Now we are ready to present our final result

THEOREM 6.1. Under the preceding assumptions, we have

$$
K_{\Phi_{\theta, n}^{1, \infty}}\left(X^{\prime}, X\right)=C_{\theta(n)}\left(X^{\prime}, X\right)
$$

with equivalent norms for all $0<\theta<1$ and $n=0, \pm 1, \pm 2, \ldots$.

Proof. We complete the proof in three steps.

At the first, we see that

$$
K_{1 / 2}^{2}\left(X^{\prime}, X\right)=H .
$$

This can be easily obtained by the equivalence $K_{1 / 2}^{2}\left(X^{\prime}, X\right)=K_{1 / 2}^{2}\left(X, X^{\prime}\right)=$ $\left(K_{1 / 2}^{2}\left(X^{\prime}, X\right)\right)^{\prime}$ and a similar argument in the proof of [7, Prop.3.2].

Next, let us assume that $0<\theta<1$ with $\theta \neq 1 / 2$ and $p=1 / \theta$, and show that

$$
K_{\theta}^{p}\left(X^{\prime}, X\right)=C_{\theta}\left(X^{\prime}, X\right) .
$$

In fact, the identities $C_{0}\left(X^{\prime}, X\right)=C_{2 \theta}\left(X^{\prime}, H\right)$ and $K_{\theta}^{p}\left(X^{\prime}, X\right)=K_{2 \theta}^{p}\left(X^{\prime}, H\right)$, for $0<\theta<1 / 2$, hold by using (6.3-4) and the reiteration. Since $X^{\prime}$ as a Banach space is of Fourier type 1, $H$ as a Hilbert space is of Fourier type 2 and $(1-2 \theta)+2 \theta / 2=1-\theta=1 / p^{\prime}$, we have $K_{2 \theta}^{p}\left(X^{\prime}, H\right) \subseteq C_{2 \theta}\left(X^{\prime}, H\right) \subseteq K_{2 \theta}^{p}\left(X^{\prime}, H\right)$ by (5.3) and hence

$$
K_{\theta}^{p^{\prime}}\left(X^{\prime}, X\right) \subseteq C_{\theta}\left(X^{\prime}, X\right) \subseteq K_{\theta}^{p}\left(X^{\prime}, X\right) .
$$

This inclusion is also valid for $1 / 2<\theta<1$ by the duality argument

$$
\left(K_{\theta}^{p}\left(X^{\prime}, X\right)\right)^{\prime} \subseteq\left(C_{\theta}\left(X^{\prime}, X\right)\right)^{\prime} \subseteq\left(K_{\theta}^{p^{\prime}}\left(X^{\prime}, X\right)\right)^{\prime} .
$$

and the identities

$$
\begin{aligned}
& \left(C_{\theta}\left(X^{\prime}, X\right)\right)^{\prime}=C^{\theta}\left(X^{\prime \prime}, X^{\prime}\right)=C_{1-\theta}\left(X^{\prime}, X\right), \\
& \left(K_{\theta}^{p}\left(X^{\prime}, X\right)\right)^{\prime}=K_{\theta}^{p^{\prime}}\left(X^{\prime \prime}, X^{\prime}\right)=K_{1-\theta}^{p^{\prime}}\left(X^{\prime}, X\right) .
\end{aligned}
$$

A similar argument yields

$$
K_{\theta}^{p}\left(X^{\prime}, X\right) \subseteq C_{\theta}\left(X^{\prime}, X\right) \subseteq K_{\theta}^{p^{\prime}}\left(X^{\prime}, X\right)
$$

for $0<\theta<1$ and $\theta \neq 1 / 2$. Thus (6.5) is immediate by combining (6.6) and (6.7).

Finally, we complete the proof for the identity $K_{\Phi_{\theta, n}^{1, \infty}}\left(X^{\prime}, X\right)=C_{\theta(n)}\left(X^{\prime}, X\right)$. For $0<\theta<1$, we choose $\theta_{0}$ such that $0<\theta_{0}<\theta<1-\theta_{0}=\theta_{1}<1$ and 
$\eta=\left(\theta-\theta_{0}\right) /\left(1-2 \theta_{0}\right)$, thus $\theta=(1-\eta) \theta_{0}+\eta \theta_{1}$. Let $\quad p_{0}=1 / \theta_{0}, p_{1}=$ $1 / \theta_{1}, 1 / q=1 / p_{0}-1 / p_{1}=\theta_{0}-\theta_{1}$, and let $1 / p=(1-\eta) / p_{0}+\eta / p_{1}=\theta$. It follows that $q\left(\theta_{1}-\theta_{0}\right)=-1$ and $q\left(\theta_{0} / p_{1}-\theta_{1} / p_{0}\right)=0$. Therefore, we conclude that

$$
\begin{aligned}
C_{\theta(n)}\left(X^{\prime}, X\right) & =C_{\eta(n)}\left(C_{\theta_{0}}\left(X^{\prime}, X\right), C_{\theta_{1}}\left(X^{\prime}, X\right)\right) & & \text { by Lemma 5.1(i) } \\
& =C_{\eta(n)}\left(K_{\theta_{0}}^{p_{0}}\left(X^{\prime}, X\right), K_{\theta_{1}}^{p_{1}}\left(X^{\prime}, X\right)\right) & & \text { by }(6.3-5) \\
& =K_{\Phi_{\theta, n}^{1, \infty}}\left(X^{\prime}, X\right) & & \text { by Lemma 5.1(ii). }
\end{aligned}
$$

ACKNOWLEDGEMENT. I appreciate several helpful conversations with Sten Kaijser.

\section{REFERENCES}

1. J. Bergh \& J. Löfström, Interpolation Spaces, Grundlehren Math. Wiss. 223, 1976.

2. Y. A. Brudnyi \& N. Y. Krugljak, Interpolation Functors and Interpolation Spaces, NorthHolland, Amsterdam, 1991.

3. M. J. Carro, J. Cerdá \& J. Soria, On Fourier type and uniform convexity of interpolation spaces, Boll. Un. Mat. Ital. 7 (1989), 883-899.

4. M. Fan, Complex interpolation functors with a family of quasi-power function parameters, Studia Math. 111 (1994), 283-305.

5. M. Fan \& S. Kaijser, Complex interpolation with derivatives of analytic functions, J. Funct Anal. 120 (1994), 380-402.

6. J. Gustavsson \& J. Peetre, Interpolation of Orlicz spaces, Studia Math. 60 (1977), 33-59.

7. U. Haagerup \& G. Pisier, Factorization of Analytic functions with values in non-commutative $L_{1}$ spaces and applications, Canad. J. Math. 41 (1989), 882-906.

8. T. Holmstedt, Interpolation of quasi-normed spaces, Math. Scand. 26 (1970), 177-199.

9. S. Kaijser, A new proof of the fundamental lemma of interpolation theory, Math. Scand. 78 (1996), 266-270.

10. J. L. Lions \& J. Peetre, Sur une classe d'espaces d'interpolation, Inst. Hautes Études Sci. Publ. Math. 19 (1964), 5-68.

11. P. Nilsson, Interpolation of Banach lattices, Studia Math. 82 (1985), 135-154.

12. J. Peetre, Sur la transformation de Fourier des fonctions à valeurs vectorielles, Rend.Sem. Mat. Univ. Padova, 42 (1969), 15-26.

SCHOOL OF ENGINEERING, DALARNA UNIVERSITY COLLEGE

S-781 88 BORLÄNGE

SWEDEN

E-mail: fmi@blg.du.se 